\title{
Resolution of Sleep Deprivation Problems using ZZZoo Pillows
}

\author{
Shunsuke Yanaka \\ Kanagawa Institute of Technology ${ }^{*}$ \\ 1030 Shimoogino, Atsugi-city, \\ Kanagawa prefecture, Japan. \\ +81-46-291-3191 \\ s1285023@cce.kanagawa-it.ac.jp
}

\author{
Motoki Ishida \\ Kanagawa Institute of Technology, Japan \\ 1030 Shimoogino, Atsugi-city, \\ Kanagawa prefecture, Japan. \\ +81-46-291-3191 \\ s0923012@cce.kanagawa-it.ac.jp
}

Takayuki Kosaka ${ }^{* 1}$

\begin{abstract}
Sleep is essential to human life. However, many people suffer from sleep deprivation, which adversely mental and physical health and results in poor productivity and even accidents. In this study, we propose the "ZZZoo Pillows" to help resolve sleep deprivation. This system comprises a huggable pillow with a built-in balloon supplied with air so that it replicates the breathing motion of a human chest. Furthermore, warm water circulating within the huggable pillow attempts to reproduce body heat, thereby giving the feeling of sleeping alongside another person. We aim to resolve sleep deprivation problems by improving emotional stability and increasing the time spent in deep sleep by providing the user with a sense of ease.
\end{abstract}

\section{Categories and Subject Descriptors}

J.3 [Computer Applications]: Life and Medical Sciences health.

\section{General Terms}

Design, Human Factors.

\section{Keywords}

Sleep deprivation, Haptics and Force Feedback, Sound sleep pillow, Breathing sensation device, Heat device.

\section{INTRODUCTION}

Sleep is essential to human life. However, many people suffer from sleep deprivation, which is believed to significantly affect blood pressure, diabetes, and immune functions [1]. Moreover, sleep deprivation not only adversely affects health but also causes deterioration of memory and poor concentration. Thus, it can lead to mental and physical illness, accidents, poor productivity, and an increase in medical expenses [2]. In 1993, the United States government noted that economic loss due to sleep-related accidents totaled 46 billion dollars a year [3]. Therefore, sleep deprivation is a serious problem that needs to be resolved.

Some general conditions of sleep include sleeping alongside

Permission to make digital or hard copies of all or part of this work for personal or classroom use is granted without fee provided that copies are not made or distributed for profit or commercial advantage and that copies bear this notice and the full citation on the first page. To copy otherwise, or republish, to post on servers or to redistribute to lists, requires prior specific permission and/or a fee.

Laval Virtual VRIC '13, March 20-22, 2013, Laval, France.

Copyright 2013 978-1-4503-1875-4 ...\$10.00 another person with skin contact, which is believed to have a physiological and psychological effect on human beings. An example of the effectiveness of skin contact is kangaroo care. It is a method of nursing low-birth-weight babies wherein the mother holds her naked baby against her chest so that their skin is in contact [4]. The physiological effects of kangaroo care improve the emotional stability of newborns and increases the time they spend in deep sleep [5]. Kangaroo care has psychological effects for both the mother and the newborn, include the formation of a good relationship and attachment between the mother and baby and a feeling of maternal expression and development as a parent.

In a study covering children form the fourth grade to high school seniors who were hospitalized, Fujii et al. [6] found high anxiety scores for children who felt anxious that they were not getting sufficient sleep. Thus, it was reported that anxiety affects the quality of sleep.

Consequently, it can be concluded that the elimination of anxiety is linked to resolving sleep deprivation. Moreover, sleeping alongside another person with skin contact and feeling another person's breathing can be expected to provide emotional stability among other benefits. Therefore, these aspects must be considered when attempting to resolve sleep deprivation problems.

It should be noted that from 2000 to 2010, the number of 20-34 year olds living with spouses and children has decreased [7]. Therefore, the spouses and children of the current generation have fewer opportunities to sleep alongside one another.

We propose ZZZoo Pillows, a system to induce sleep, for resolving the above-mentioned issues. This system comprises a huggable pillow that attempts to reproduce the conditions of breathing and body heat felt when sleeping alongside another person. The huggable pillow repeatedly expands and contracts in a manner similar to the motion of the human chest during breathing and is able to reproduce human warmth.

\section{SYSTEM CONFIGURATION}

The ZZZoo Pillow system comprises a breathing sensation device, which reproduces a person's breathing conditions by using a balloon inside the huggable pillow, and a heating device, which reproduces the body heat of a person using warm water (Fig. 1).

The built-in balloon attempts to replicate the expansion of the human chest. Air flow from an air compressor is regulated by an electromagnetic valve and an Arduino microcontroller.

The breathing rate used in this system is based on a study by Furuya et al. [8], who experimentally discovered that the lesser 
the time taken to fall asleep, the longer and more efficient the sleep. The study indicated that when the subject took less time to fall asleep, the recorded respiratory rate during sleep was higher (16 breaths per minute). Moreover, the respiratory rate results were constant 30 minutes after the subject had fallen asleep.

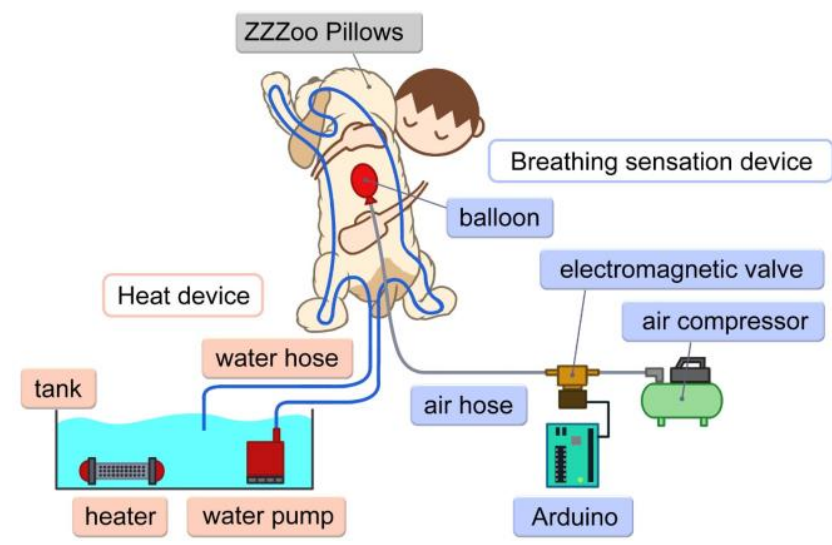

Figure 1. Schematic of ZZZoo Pillow system.

Therefore, we inflated the balloon in the huggable pillow at a rate of 16 breaths per minute.

The water in the tank is heated to approximately $45^{\circ} \mathrm{C}$. The body heat of a person is reproduced by this warm water, which is drawn from the water pump and circulated using a hose running inside the huggable pillow. Water is circulated at approximately $45^{\circ} \mathrm{C}$, which is higher than a person's body temperature, so the user can feel the warmth even through the fabric of the huggable pillow.

\section{EVALUATION AND CONSIDERATIONS}

Approximately 300 people tried the pillow at an exhibit(Fig. 2). After they had used the pillow, we conducted a survey and obtained responses from 84 people ( 40 men and 44 women).

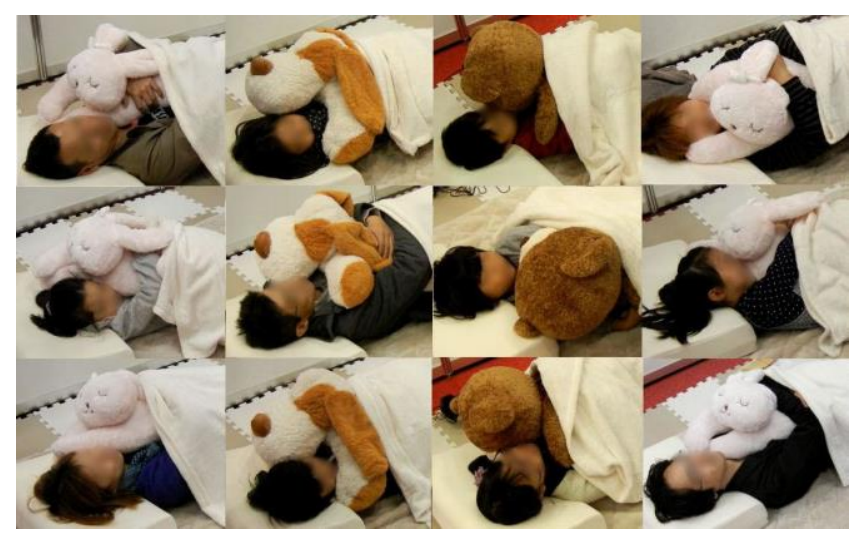

Figure 2. ZZZoo Pillows exhibit

When the users were asked "Do you feel that the breathing conditions replicated by the huggable pillow would induce sleep?" $61 \%$ responded with "I feel it would."

Next, when asked "Do you feel that the fact the huggable pillow is heated to reproduce a person's body heat would encourage sleep?" $85 \%$ responded with "I feel it would."

Finally when asked "Would you like to use this system in the future?" the majority of the users responded with "I would like to use it" (Fig. 3).
All the people who used the pillow and responded with "I would not like to use it" had responded to earlier questions stating that they did not currently suffer from sleep deprivation. Furthermore, almost all users responded that they can usually fall asleep easily. This implies that the reason some users did not want to use the system in the future was that they were currently not suffering from any sleep-related problems and hence did not need the system.

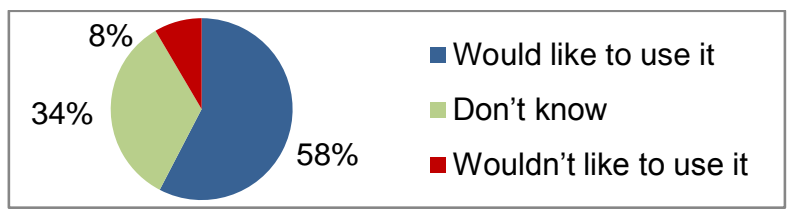

Figure 3. User response to the question "Would you like to use this system in the future?"

\section{CONCLUSION}

We developed the "ZZZoo Pillows" to help resolve sleep deprivation problems. This system attempts to provide a sense of ease and ultimately contribute to the resolution of sleep deprivation by reproducing the breathing motion and body heat felt when a person is sleeping alongside another.

According to subjective evaluation, reproducing a person's breathing and body heat using a huggable pillow helps induce sleep. In future, we plan to verify the sleep-inducing effect of the system using physiological reports of users. We will also perform additional subjective evalutions.

\section{REFERENCES}

[1] Komaba, Y., and Inoue, Y. 2007. The Impact of Sleep Disorders on Social Life. In Psychosomatic Medicine. 47, 9(Sep. 2007), 785-791.

[2] Song, Y., and Nishino, S. 2008. National Efforts in America to Reduce Societal Loss due to Sleep Disorders and the Outcome of these Efforts. In University of Occupational \& Environmental Health Magazine. 30, 3(Sep. 2008), 329-352.

[3] Dement WC. 1993. Wake up America : A National Sleep Alert Executive Summary 1. The National Commission on Sleep Disorders Research, 1-75.

[4] Oishi, M., Asada, S., Kuroki, E., Date, K., Miyama, C., and Nakao, Y. 2006. Kangaroo Care in Japan Based on Literature. In Health Science Research. 19, 1(2006), 21-26.

[5] Shima, R., Niwakawa, H., Hirano, Y., Tanuma, K., Ikeda, N., and Kinoshita, K. 2003. Study of Kangaroo Care Immediately After Birth. In Japan Society of Maternal Health. 44, 4(Dec. 2003), 488-494.

[6] Fujii, K., Kusaba, H., and Nomuguchi, C. 2007. Sleep in Hospitalized Adolescents: Relationship between the Characteristics of their Sleep and Anxiety. In The South Kyushu Journal of Nursing. 5, 1(Mar. 2007), 29-36.

[7] Iwai, N. 2011. The Current Picture and Overall Trends of the Japanese Family Based on JGSS Cumulative Data from 2000 to 2010. In Japan Society of Family Sociology. 23, 1(2011), $30-42$.

[8] Furuya, Y., Kanamori, N., Fujiwara, Y., and Tanaka, H. 2011. Study of Thoracic Breathing Patterns and the Process of Falling Asleep. In Journal of Physiological Psychology and Psychophysiology. 29, 2(Aug. 2011), 95. 